Sharif University of Technology
Scientia Iranica
SCIENTIA
IRANICA

\title{
Generalized heat transfer and entropy generation of stratified air-water flow in entrance of a mini-channel
}

\author{
N. Zehtabiyan-Rezaie ${ }^{\mathrm{a}}$, S. Rahimi Damirchi-Darasi ${ }^{\mathrm{b}}$, M.H. Fazel Zarandi ${ }^{\mathrm{b}, \mathrm{c}}$ \\ and M. Saffar-Avval ${ }^{\mathrm{a}, \mathrm{d}, *}$ \\ a. Department of Mechanical Engineering, Amirkabir University of Technology, Tehran, Iran. \\ b. Department of Industrial Engineering, Amirkabir University of Technology, Tehran, Iran. \\ c. Laboratory of Knowledge Intelligent System, University of Toronto, Toronto, Canada. \\ d. Energy and Control Center of Excellence, Amirkabir University of Technology, Tehran, P.O. Box 1591-634311, Iran.
}

Received 31 October 2015; received in revised form 26 August 2016; accepted 1 October 2016

\section{KEYWORDS}

Stratified two-phase

flow;

Fuzzy inference

system;

Heat transfer;

Entropy generation;

Mini-channel.

\begin{abstract}
In the present study, a rule-based fuzzy inference system is used to predict heat transfer and entropy generation of stratified air-water flow in horizontal mini-channel as a function of a wide range of important parameters. Numerical data of our recent study are used to develop and test the system. The GK clustering algorithm is used to cluster the data. Fuzzy rules are generated based on the Sugeno-Yasukawa algorithm by using trapezoidal membership functions. The FATI and FITA approaches are implemented in the inference engine and, finally, the combination of the two approaches is defuzzified. The Mamdani and logical methods with the Yager operators are used and unified in both approaches. The parametric form of the system is a feature of the present study, which can be used as an effective tool to improve the accuracy of the results. The novelty of the present study is the presentation of the generalized diagrams for the developing region of the channel, which seems to be useful for engineering applications. In addition, generalized diagrams of average Nusselt numbers as well as total entropy generation can identify the appropriate ranges for the volumetric flow rate ratio and the Reynolds number.
\end{abstract}

(C) 2017 Sharif University of Technology. All rights reserved.

\section{Introduction}

In stratified two-phase flows, which occur in many industrial and scientific applications, two fluids move in the same direction in low flow rates, creating a free interface between the upper and lower phases [14]. Free interface modeling is a complex step of the studies dealing with two-phase flows; therefore, extra computations are required to handle the interface in addition to solve the governing equations such as the continuity, momentum, and energy equations.

*. Corresponding author. Fax: +982166419736 E-mail address: mavval @aut.ac.ir (M. Saffar-Avval)
Here, some of the studies relevant to the smooth stratified two-phase flow in channels are reviewed. Hansen and Vested [5] developed a two-dimensional model to study smooth and wavy stratified two-phase flows in a horizontal channel while the free interface was assumed to be moving wall. In addition, the velocity profiles of the phases were composed of two logarithmic functions. Their model showed a good accuracy in the smooth stratified flow in comparison with experimental data. Yap et al. [6] studied smooth gas-liquid and vapor-liquid flows in channel by using the level set method to capture the interface. They implemented a mass correction term to overcome the level set method problems satisfying the continuity. Datta et al. [7] performed a numerical study to model two-phase flow in a plane channel and a square duct by using the 
level set method. In addition, they presented analytical solutions for the fully developed region of the domain in which the governing equations could be simplified. They examined the effects of several parameters such as viscosity ratio of the phases and the Reynolds number while the densities of the phases were assumed to be equal. Gada et al. [8] investigated the heat transfer of stratified flow of two immiscible fluids in a plane channel by implementing the level set method besides the momentum and energy equations. An analytical solution was also proposed for the fully developed region of the channel. Zehtabian-Rezaie et al. [9] performed a numerical study in the field of flow, heat transfer, and entropy generation of developing liquid-liquid and gas-liquid stratified two-phase flows in mini-channel. They used the Volume Of Fluid method (VOF) to model the free interface between the upper and lower phases. In addition, they presented analytical solutions for the momentum, energy, and entropy generation equations in the fully developed region of the channel. They presented generalized diagrams for the pressure gradient, interface height, and upper and lower Nusselt numbers in the fully developed region as functions of important parameters. According to the literature review, it seems that no generalized diagrams have been provided in any studies to present the material about the developing region of the stratified two-phase flow. Most of the numerical results of the studies present limited data, which are obtained by execution of the developed codes for specific cases. It seems that there is a large demand for the studies presenting generalized diagrams to simplify the results of the studies by other researchers and engineers. As a result of the complexity of the numerical studies as well as the high cost of experiments, artificial intelligence can play a vital role in generation of the outputs for the cases that cannot be experimentally performed or numerically calculated. Application of artificial intelligence in fluid flow and heat transfer prediction has been performed in several studies [10-14]. It seems that entropy generation analysis of stratified gas-liquid flow is not extensively considered in the literature; however, multiple studies focus on entropy generation in liquid flow $[15,16]$.

The main purpose of this paper is to complete our earlier work [9] by presenting generalized diagrams for the flow, heat transfer, and entropy generation characteristics in the developing region of the smooth stratified air-water flow, which is done for the first time in the literature to the best knowledge of the authors. A fuzzy inference system based on the extensive numerical data obtained from our recent study [9] is developed to predict the desirable results in the format of generalized diagrams without imposing high computational cost. Presentation of the generalized diagrams for interface height, maximum axial velocity, and upper and lower Nusselt numbers in the developing region as well as average upper and lower Nusselt numbers and total entropy generation in the whole channel for a wide range of flow conditions such as volumetric flow rate ratio and the Reynolds number facilitates the application of the results by other researchers. The Gustafson and Kessel clustering algorithm [17] is used to cluster the data as the first step of the fuzzy inference system development. Fuzzy rules are generated based on the Sugeno-Yasukawa algorithm [18] by using the trapezoidal membership functions. The FATI (First Aggregate Then Infer) and FITA (First Infer Then Aggregate) approaches are implemented in the inference engine and, finally, the combination of the two approaches is defuzzified. The Mamdani and logical methods with the Yager operators are used and unified in both approaches. The parametric form of the fuzzy inference system is another feature of the present study, which can be used as an effective tool to improve the accuracy of the results.

\section{Problem description}

The schematic illustration of the problem is shown in Figure 1. Upper and lower fluids, which are assumed to be incompressible fluids with constant thermo-physical properties, flow into the mini-channel with uniform velocity and temperature. The ratio of the volumetric flow rate of the upper fluid to the volumetric flow rate of the lower fluid at the channel inlet is named volumetric flow rate ratio, which is an important parameter in the stratified two-phase flows. Constant heat fluxes are applied on the upper and lower walls. The height of the free interface constructed between the phases varies in the developing region of the channel. In the developing region, the axial velocity profile varies in the $x$-direction as well. As the hydro-dynamically fully developed conditions occur, the interface height remains fixed [9].

Non-dimensional forms of the governing equations including the continuity, momentum, energy, and entropy generation equations are presented in Eqs. (1) to $(5)$ :

- Continuity:

$$
\nabla \cdot \vec{V}=0
$$

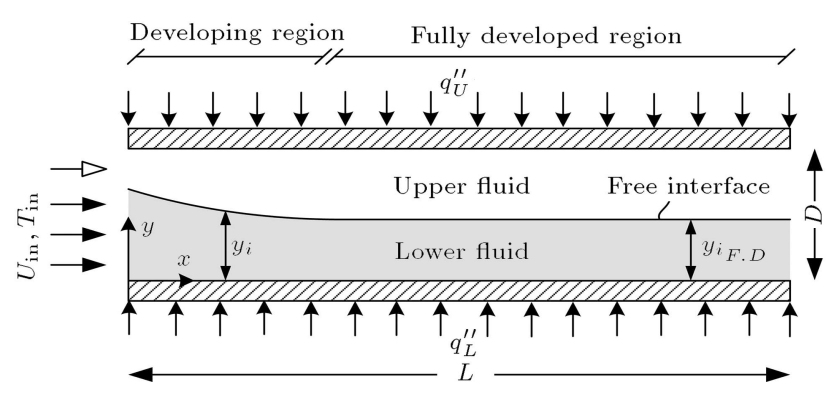

Figure 1. Schematic view of the problem [9]. 
- Momentum:

$$
\begin{aligned}
\rho \frac{\partial u}{\partial t} & +\rho \frac{\partial\left(u^{2}\right)}{\partial x}+\rho \frac{\partial(u v)}{\partial y}=-\frac{\partial P}{\partial x} \\
& -\frac{1}{\operatorname{Re}}\left[\frac{\partial\left(-2 \mu \frac{\partial u}{\partial x}\right)}{\partial x}+\frac{\partial\left(-\mu\left(\frac{\partial v}{\partial x}+\frac{\partial u}{\partial y}\right)\right)}{\partial y}\right] \\
\rho \frac{\partial v}{\partial t} & +\rho \frac{\partial(u v)}{\partial x}+\rho \frac{\partial\left(v^{2}\right)}{\partial y}=-\frac{\partial P}{\partial y} \\
& -\frac{1}{\operatorname{Re}}\left[\frac{\partial\left(-\mu\left(\frac{\partial v}{\partial x}+\frac{\partial u}{\partial y}\right)\right)}{\partial x}+\frac{\partial\left(-2 \mu \frac{\partial v}{\partial y}\right)}{\partial y}\right]
\end{aligned}
$$

- Energy:

$$
\begin{aligned}
& \rho c_{p}\left(\frac{\partial T}{\partial t}+\frac{\partial(u T)}{\partial x}+\frac{\partial(v T)}{\partial y}\right) \\
& =\frac{1}{\operatorname{Re} \cdot \operatorname{Pr}_{L}}\left[\frac{\partial\left(k \frac{\partial T}{\partial x}\right)}{\partial x}+\frac{\partial\left(k \frac{\partial T}{\partial y}\right)}{\partial y}\right]
\end{aligned}
$$

- Entropy generation [19]:

$$
\begin{aligned}
S_{\text {gen }}= & \frac{k}{(T+\delta)^{2}}\left[\left(\frac{\partial T}{\partial x}\right)^{2}+\left(\frac{\partial T}{\partial y}\right)^{2}\right] \\
& +\left(\operatorname{Pr}_{L} \cdot \xi\right)\left(\frac{\mu \cdot \delta}{T+\delta}\right)\left\{2\left[\left(\frac{\partial u}{\partial x}\right)^{2}+\left(\frac{\partial v}{\partial y}\right)^{2}\right]\right. \\
& \left.+\left(\frac{\partial u}{\partial y}+\frac{\partial v}{\partial x}\right)^{2}\right\} .
\end{aligned}
$$

The dimensionless parameters of Eq. (6) are used to derive the aforementioned equations:

$$
\begin{aligned}
& x=\frac{x^{\prime}}{D} ; \quad y=\frac{y^{\prime}}{D}, \\
& t=\frac{u_{\text {in }} \cdot t^{\prime}}{D}, \quad P=\frac{P^{\prime}-P_{\mathrm{in}}}{\rho_{L} \cdot u_{\mathrm{in}}^{2}}, \quad u=\frac{u^{\prime}}{u_{\mathrm{in}}} \\
& v=\frac{v^{\prime}}{u_{\mathrm{in}}}, \quad T=k_{L} \frac{T^{\prime}-T_{\mathrm{in}}}{q_{L}^{\prime \prime} \cdot D}, \\
& S_{\text {gen }}=\frac{S_{\text {gen }}^{\prime \prime \prime} \cdot D^{2}}{k_{L}}, \quad \rho=\frac{\rho^{\prime}}{\rho_{L}} ; \\
& \mu=\frac{\mu^{\prime}}{\mu_{L}} ; \quad k=\frac{k^{\prime}}{k_{L}} ; \quad c_{p}=\frac{c_{p}^{\prime}}{c_{p_{L}}} \\
& \operatorname{Re}=\frac{\rho_{L} \cdot u_{\mathrm{in}} \cdot D}{\mu_{L}} ; \quad \operatorname{Pr}_{L}=\frac{\nu_{L}}{\alpha_{L}} ; \\
& \delta=\frac{k_{L} \cdot T_{\mathrm{in}}}{q_{L}^{\prime \prime} \cdot D} ; \quad \xi=\frac{u_{\mathrm{in}}^{2}}{c_{p_{L}} \cdot T_{\mathrm{in}}}
\end{aligned}
$$

where $D$ and $L$ are height and length of the minichannel, respectively; $u_{\text {in }}$ is the axial inlet velocity; $P$ is the dimensionless pressure; $u$ and $v$ are the dimensionless velocities in $x$ and $y$ directions; $T$ is the dimensionless temperature; $S_{\text {gen }}$ is dimensionless entropy generation; $\rho, \mu, k$, and $c_{p}$ are the dimensionless density, viscosity, thermal conductivity, and constantpressure specific heat, respectively; and $\operatorname{Re}, \operatorname{Pr}_{L}, \delta$, and $\xi$ are the Reynolds number, Prandtl number of the lower fluid, and the two dimensionless parameters used in entropy generation equation [9].

\section{Numerical data used}

The numerical data used in the present study were extracted from the recent study by the same authors [9] in which the aforementioned equations were solved numerically and analytically. A fully staggered grid was utilized to solve the continuity and Navier-Stokes equations by using the second-order finite difference method based on the projection algorithm proposed by Chorin [20]. The volume of fluid method was implemented to reconstruct the interface through the advection equation (Eq. (7)), which updates the volume fraction field in each time step using the velocity field [21-25]. Important parameters of the study are presented in Table 1 . The recent study by ZehtabiyanRezaie et al. [9] can be employed to generate large amount of data to develop a fuzzy inference system. Then, the fuzzy inference system can be used to predict the two-phase flow characteristics in the developing region as generalized charts. The algorithm of the numerical method in Zehtabiyan-Rezaie et al.'s study [9] is presented in Figure 2.

$$
\frac{\partial f}{\partial t}+\nabla \cdot(f V)-f \nabla \cdot V=0.0
$$

Table 1. Important parameters of the study [9].

\begin{tabular}{ccc}
\hline Parameter & Unit & Value \\
\hline$D$ & $\mathrm{~m}$ & 0.001 \\
$L$ & $\mathrm{~m}$ & 0.01 \\
$\rho_{r}$ & - & 0.1160 \\
$\mu_{r}$ & - & 0.0218 \\
$k_{r}$ & - & 0.0426 \\
$c_{p_{L}}$ & - & 0.2417 \\
$\operatorname{Pr}_{L}$ & - & 5.8890 \\
$q_{L}^{\prime \prime}, q_{U}^{\prime \prime}$ & $\mathrm{W} / \mathrm{m}^{2}$ & 1000000.0 \\
$\rho_{L}$ & $\mathrm{~kg} / \mathrm{m}^{3}$ & 1001.0 \\
$\mu_{L}$ & $\mathrm{~kg} /(\mathrm{m} . \mathrm{s})$ & 0.0008527 \\
$k_{L}$ & $\mathrm{~W} /(\mathrm{m} . \mathrm{K})$ & 0.6019 \\
$c_{p_{L}}$ & $\mathrm{~J} /(\mathrm{kg} . \mathrm{K})$ & 4156.0 \\
$T_{\mathrm{in}}$ & $\mathrm{K}$ & 300.0 \\
\hline
\end{tabular}




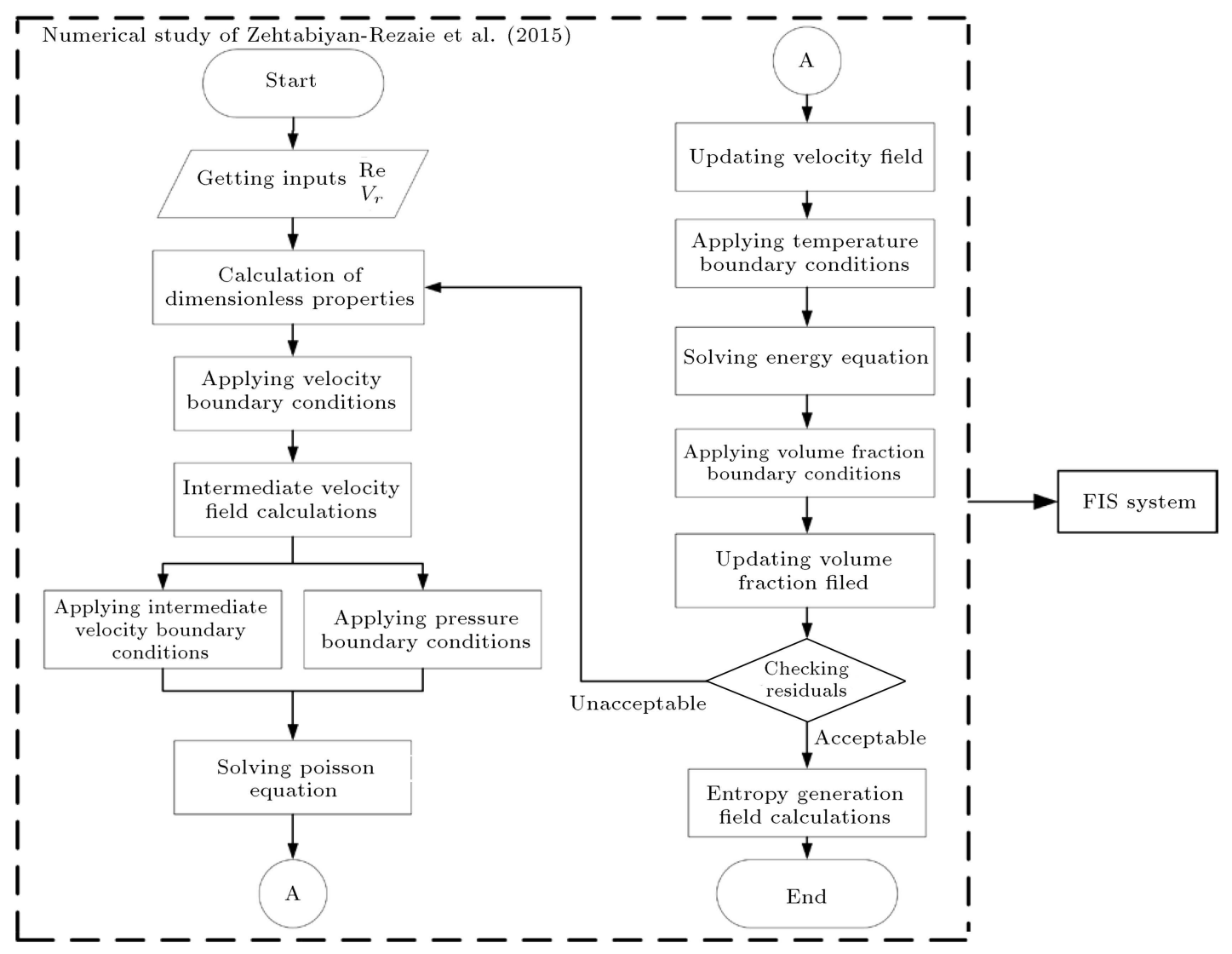

Figure 2. Algorithm of the numerical study of Zehtabiyan-Rezaie et al. [9] .

\section{Fuzzy Inference System (FIS) development}

Before selecting the fuzzy logic as a tool to handle the problem, a semantic analysis of the problem must be performed to find the most appropriate method. In the present problem, extensive output results are generated through the Fortran code developed for the earlier study of the authors [9]; it seems that the amount of data as well as the dependencies between the inputs and outputs leaves no other way but using fuzzy logic, which was proposed by Zadeh [26] for the first time in 1965. In addition, since the fuzzy intelligence systems have no presupposition about the statistical distribution of the result, it may give good outputs in comparison with the common statistical methods [13].

As shown in Figure 3, the main objective of the fuzzy inference system development in the present study is prediction of the interface height, maximum axial velocity, and upper and lower Nusselt numbers of stratified air-water flow in the developing region of the mini-channel as well as the average upper and lower Nusselt numbers and the total entropy generation in the whole channel as functions of the volumetric flow rate ratio and the Reynolds number.

\subsection{Clustering the data}

The first step of the system development is clustering of the data. Gustafson and Kessel algorithm [17] is selected to cluster the data and find the cluster membership function matrix. The objective function of the GK algorithm is given in Eq. (8):

$$
J_{G K}^{m}(U, A, V, X)=\sum_{i=1}^{c} \sum_{k=1}^{N} u_{i k}^{m} d\left(x_{k}, v_{i}\right) .
$$

The main steps of the algorithm are as follows, which are extensively explained by Fazel Zarandi et al. [27]:

- Step 1: Compute cluster prototypes:

$$
v_{i}^{(l)}=\frac{\sum_{k=1}^{N}\left(u_{i k}^{(l-1)}\right)^{m} x_{k}}{\sum_{k=1}^{N}\left(u_{i k}^{(l-1)}\right)^{m}} .
$$

- Step 2: Compute the cluster covariance matrices:

$$
F_{i}=\frac{\sum_{k=1}^{N}\left(u_{i k}^{(l-1)}\right)^{m}\left(x_{k}-v_{i}^{(l)}\right)\left(x_{k}-v_{i}^{(l)}\right)^{T}}{\sum_{k=1}^{N}\left(u_{i k}^{(l-1)}\right)^{m}} .
$$

- Step 3: Compute the distances:

$$
D_{i k A_{i}}^{2}=\left(x_{k}-v_{i}^{(l)}\right)^{T}\left[\rho_{i} \operatorname{det}\left(F_{i}\right)^{1 / n} F_{i}^{-1}\right]\left(x_{k}-v_{i}^{(l)}\right) \text {. }
$$

- Step 4: Update the partition matrix: 


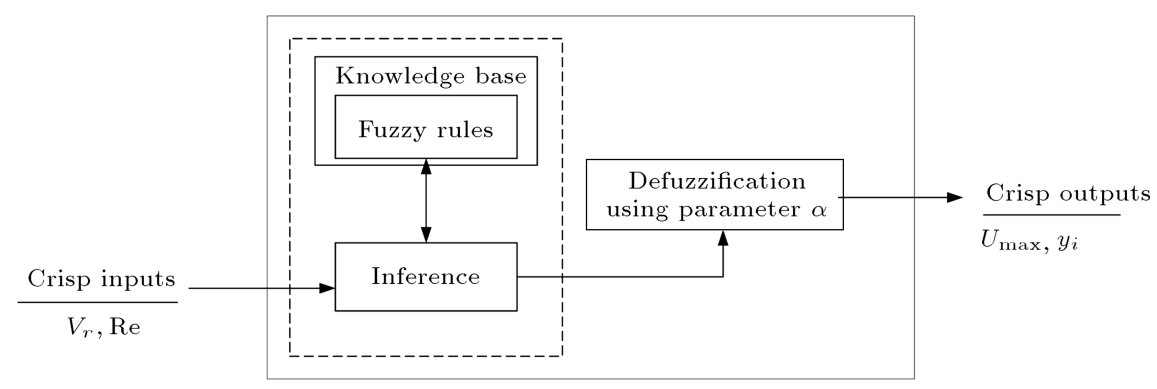

Figure 3. Fuzzy inference system.

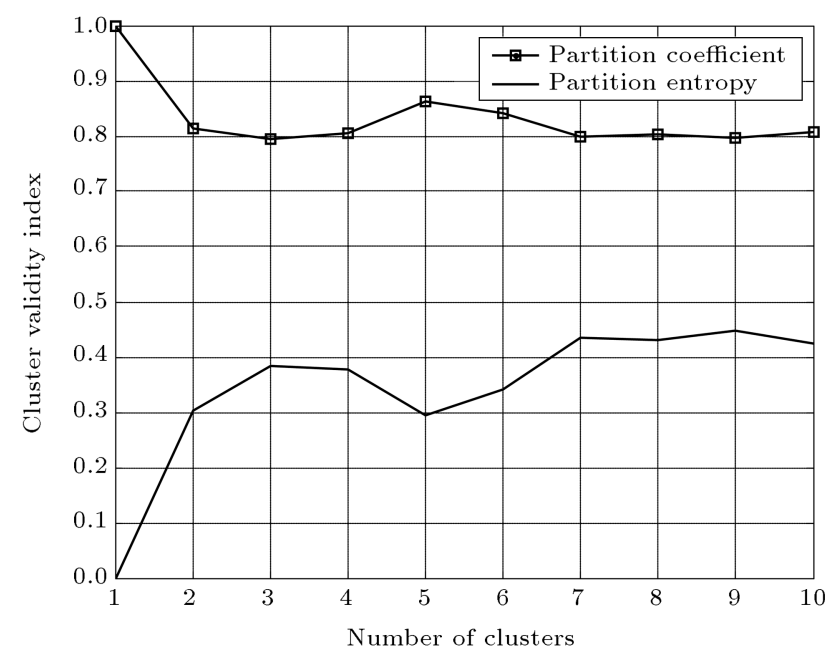

Figure 4. Validity indices as a function of cluster numbers in GK method for a specific data set.

$$
u_{i k}^{(l)}=\frac{1}{\sum_{j=1}^{c}\left(D_{i k A_{j}} / D_{i k A_{i}}\right)^{\frac{2}{m-1}}}
$$

In the present study, the value of weighting exponent $(m)$ is assumed to be equal to 2 . In order to find the appropriate number of clusters, several cluster validity indices are proposed. In this study, the partition coefficient and partition entropy validity indices are utilized, which are formulated by Bezdek [28]. In Figure 4, two cluster validity indices are plotted versus number of clusters for one of the data sets of the numerical data. It can be seen that selection of 5 clusters for that set can satisfy both of the indices.

\subsection{Extraction of the rules}

To this step, the clusters are generated using the GK method [17]. By transferring the output membership functions into the input space and projecting the fuzzy cluster onto the $X_{1}\left(V_{r}\right)$ and $X_{2}(\mathrm{Re})$ spaces, the membership functions of the inputs as well as the outputs are produced [18] and the fuzzy set is approximated with trapezoidal type membership functions as shown for one of the data sets in Figure 5.

\subsection{Inferring with parametric operators and FATI-FITA combination}

As illustrated in Figure 6, the Yager parametric operators are used as t-norms and s-norms. The FATI and FITA approaches are implemented in inference module, each utilizing the Mamdani's logical and unified method. Finally, a combination of the FATI and FITA approaches is computed as the final output. The FATI and FITA approaches are combined through Eq. (13).

$$
\mathrm{Comb}_{\mathrm{FATI}-\mathrm{FITA}}=\mathrm{FATI} \cdot(1-\zeta)+\mathrm{FITA} \cdot \zeta .
$$

At the end, defuzzifying is performed by using Eq. (14); in this equation, the parameter $\alpha$ generalizes the formula. In this problem, $\alpha$ (defuzzifying parameter), $\beta$ (Mamdani-Logical combination parameter), $\zeta$ (FATIFITA combination parameter), and $\omega$ (Yager operators parameter) are the parameters that must be tuned:

$$
y^{*}=\frac{\int_{y_{0}}^{y \bullet} y\left[\mu_{F}(y)\right]^{\alpha} d y}{\int_{y_{0}}^{y}\left[\mu_{F}(y)\right]^{\alpha} d y} .
$$

\section{Results and discussion}

The numerical data were divided into two groups; $75 \%$ of the whole data were used to construct the system and $25 \%$ were used to test it and tune the parameters. In order to evaluate the system, 3 cases were used, which were not among the system development and testing data. In Figure 7, developing of interface height, maximum axial velocity, and upper and lower Nusselt numbers extracted from FIS is compared with the results of CFD study [9]. It seems that the FIS results show good accuracy and the developed system can be utilized to achieve the aims of the study.

\subsection{Interface height in the developing region}

In Figure 8, the developing interface height is presented as a function of the volumetric flow rate ratio and the Reynolds number. Decreasing the Reynolds number lessens the development length. In the air-water stratified two-phase flow, the maximum velocity is located 

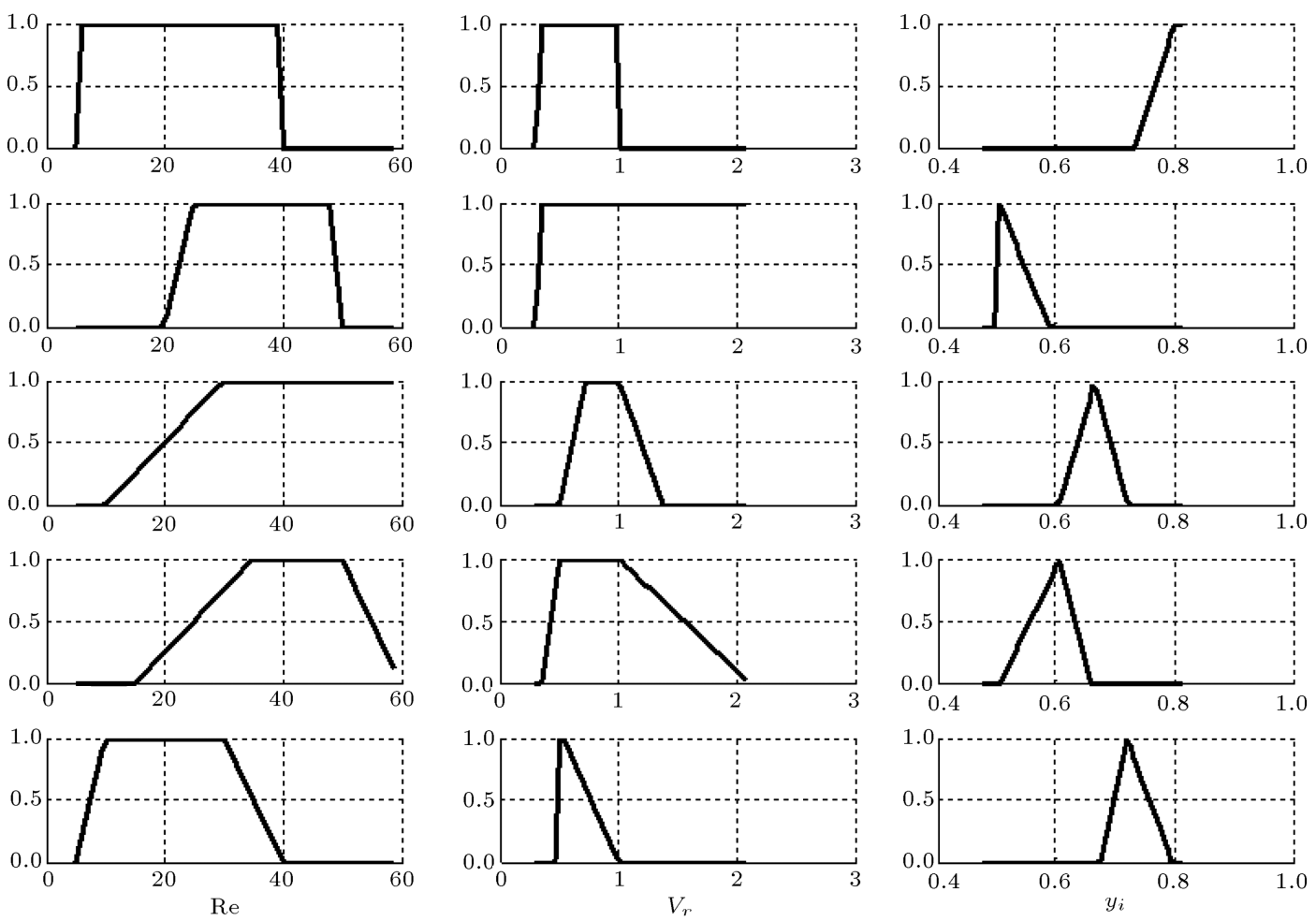

Figure 5. Membership functions of the Re, $V_{r}$ (inputs) and $y_{i}$ (outputs) for a specific data set.

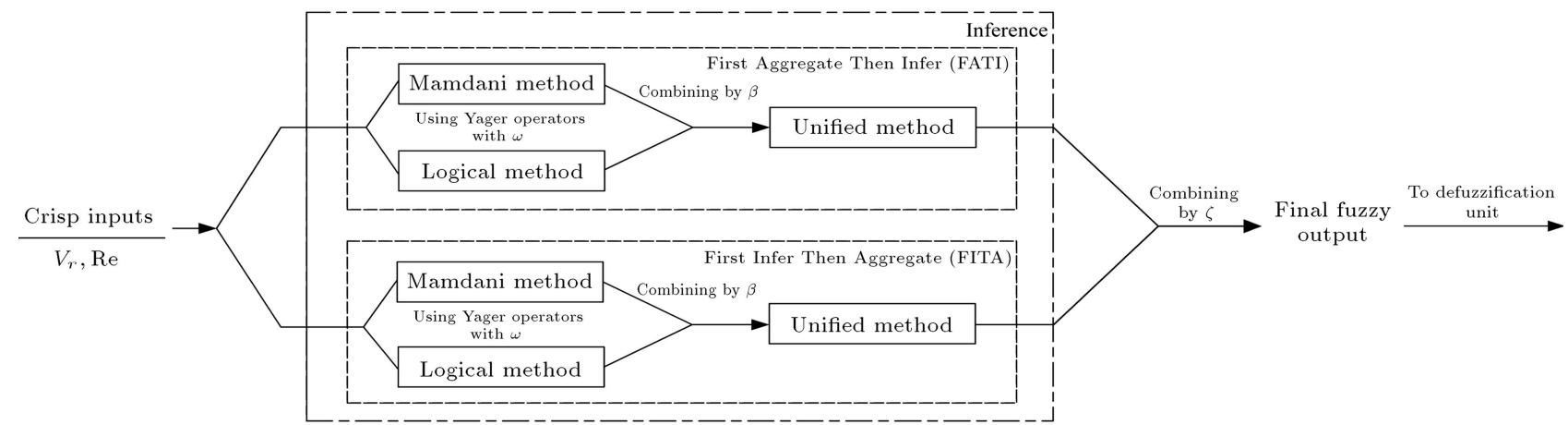

Figure 6. Inference module of the developed FIS.

in the upper fluid (air); therefore, the conservation of mass forces the interface to move towards the upper wall until the fully developed conditions are achieved.

\subsection{Maximum axial velocity in the developing region}

In Figure 9, the generalized diagram of the developing maximum axial velocity is shown. It is evident that for the volumetric flow rate ratios less than 0.5 at $x=3$ and 4 , fully developed conditions are achieved; therefore, the interface height is nearly independent of the Reynolds number.

\subsection{Heat transfer coefficient in the developing region}

The total bulk temperature and the local Nusselt numbers that are defined at the upper and lower walls are calculated by using Eqs. (15) and (16) [9]:

$$
\begin{aligned}
& T_{\mathrm{bulk}}=\int_{0}^{1} \rho c_{p} u T d y / \int_{0}^{1} \rho c_{p} u d y \\
& \mathrm{Nu}_{U}=h_{U} \frac{D}{k_{U}}=\frac{\left(\frac{q_{r}^{\prime \prime}}{k_{r}}\right)}{\left(T_{\mathrm{wall}_{U}}-T_{\mathrm{bulk}}\right)} \\
& \mathrm{Nu}_{L}=h_{L} \frac{D}{k_{L}}=\frac{1.0}{\left(T_{\mathrm{wall}_{L}}-T_{\mathrm{bulk}}\right)}
\end{aligned}
$$

In Figure 10, the upper Nusselt number is presented for wide ranges of the Reynolds and volumetric flow rate ratio. The upper Nusselt number decreases along the developing region of the mini-channel. By increasing the Reynolds number, the fully developed conditions 


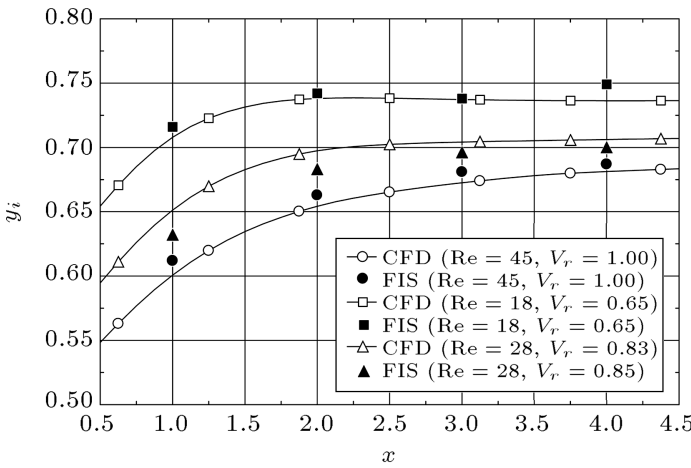

(a)

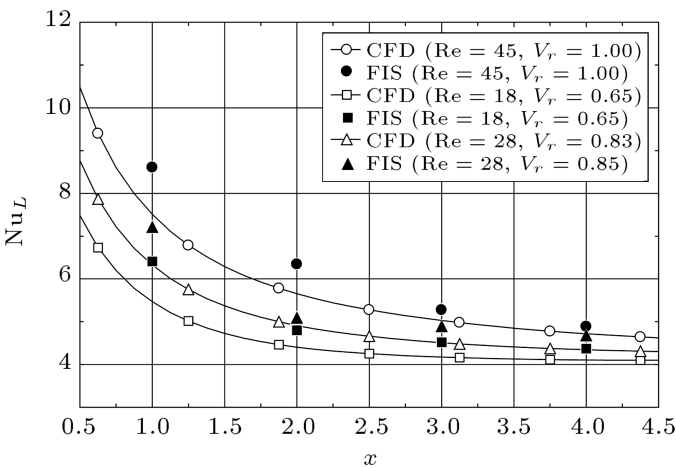

(c)

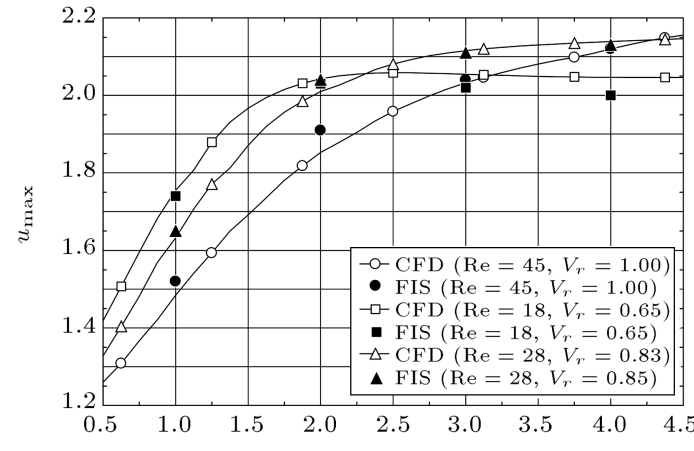

(b)

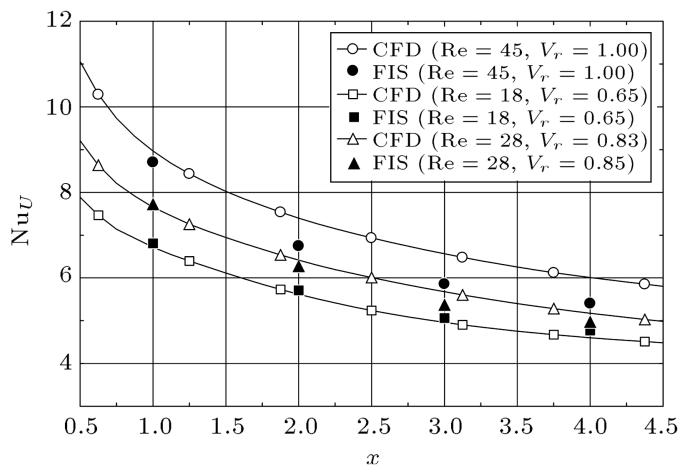

(d)

Figure 7. Validation of the FIS with the numerical results of Zehtabiyan-Rezaie et al. [9]: (a) Local interface height in the developing region, (b) local maximum axial velocity in the developing region, (c) local lower Nusselt number in the developing region, and (d) local upper Nusselt number in the developing region.

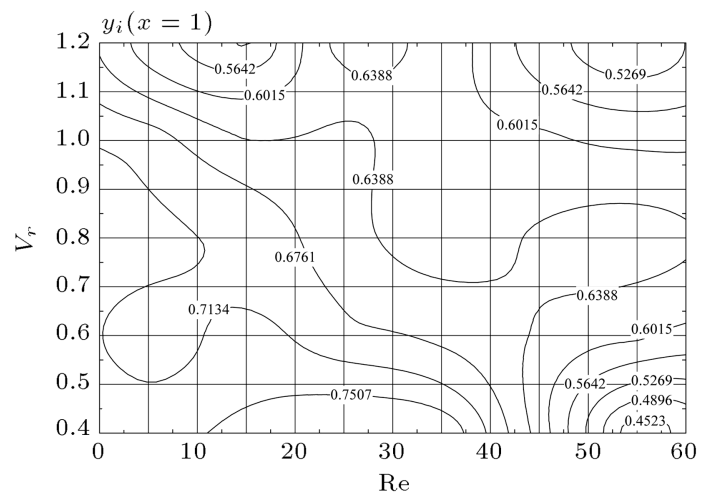

(a)

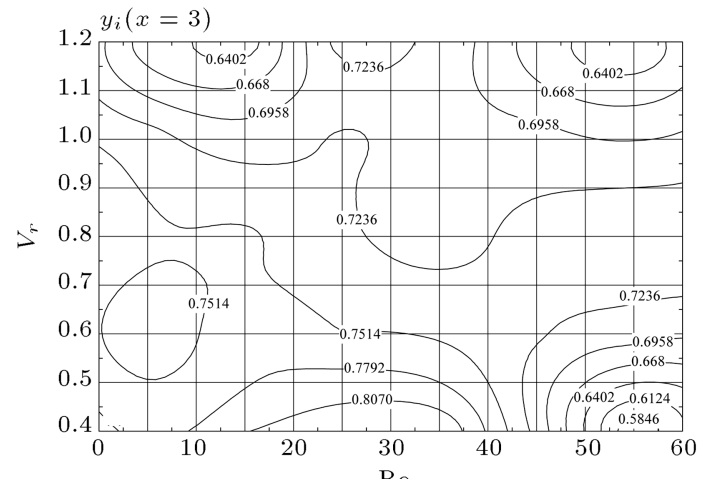

(c)

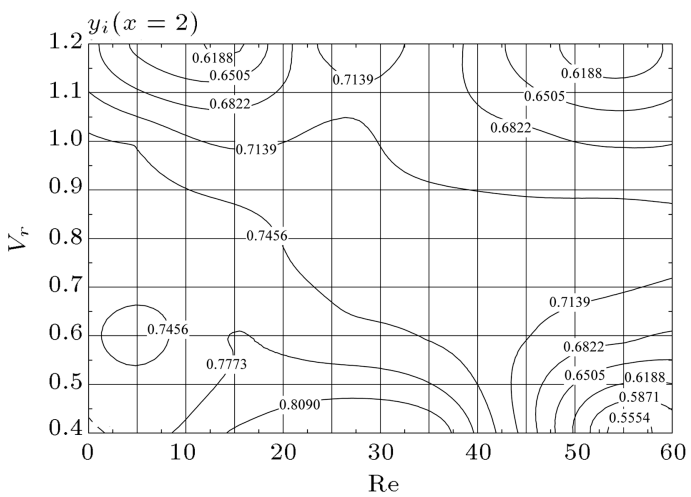

(b)

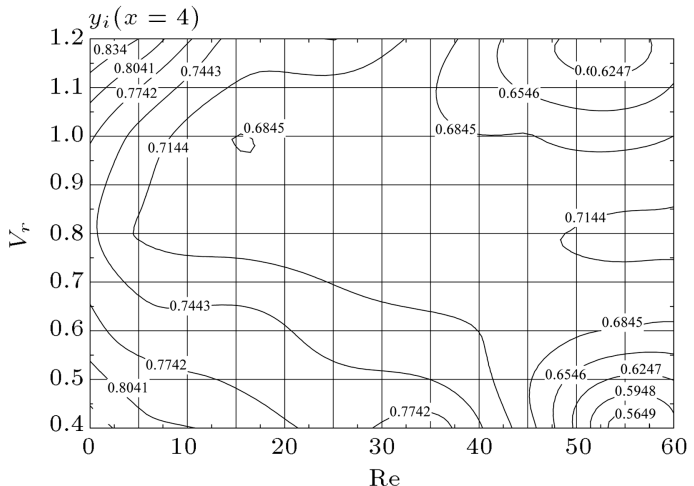

(d)

Figure 8. Generalized diagram of developing interface height of stratified air-water flow as a function of the volumetric flow rate ratio and Reynolds number at $x=1,2,3$, and 4 from channel inlet. 


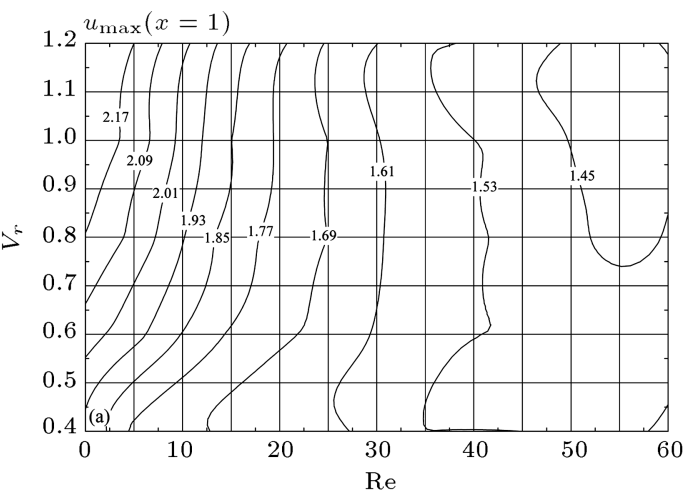

(a)

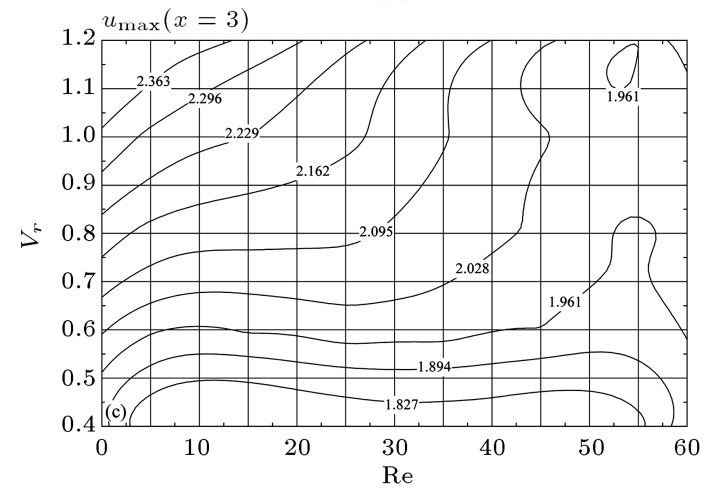

(c)

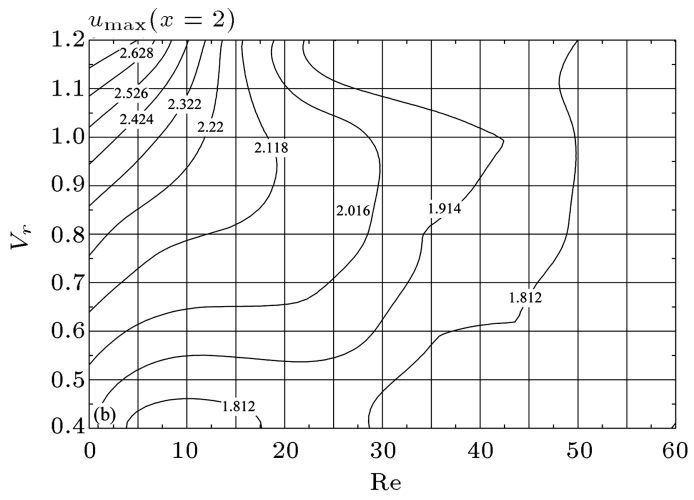

(b)

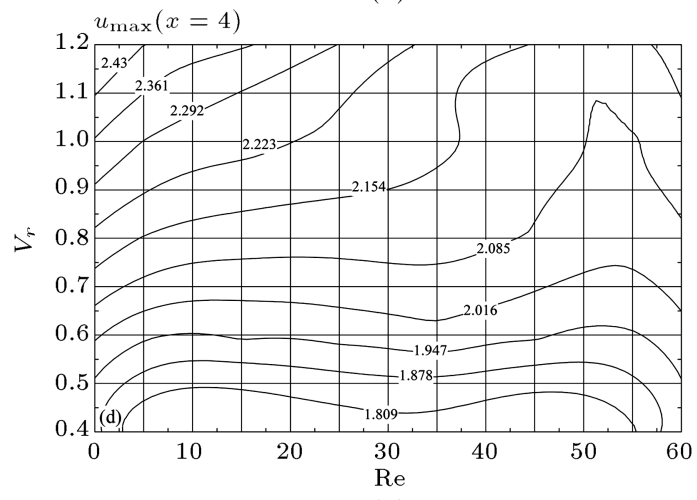

(d)

Figure 9. Generalized diagram of developing maximum axial velocity of stratified air-water flow as a function of the volumetric flow rate ratio and Reynolds number at $x=1,2,3$, and 4 from channel inlet.

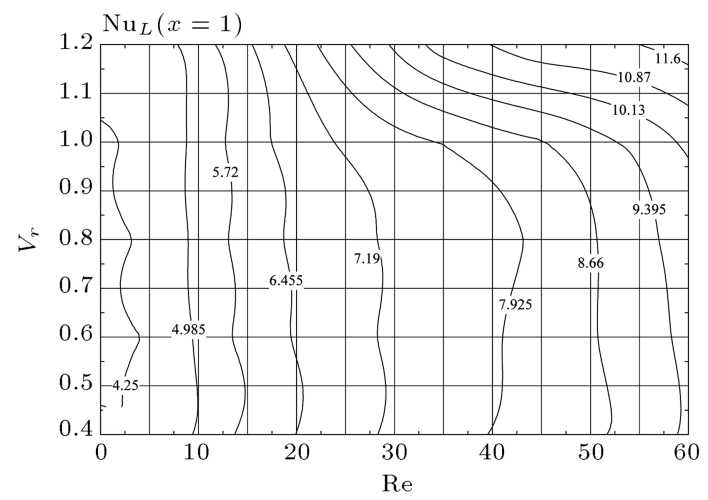

(a)

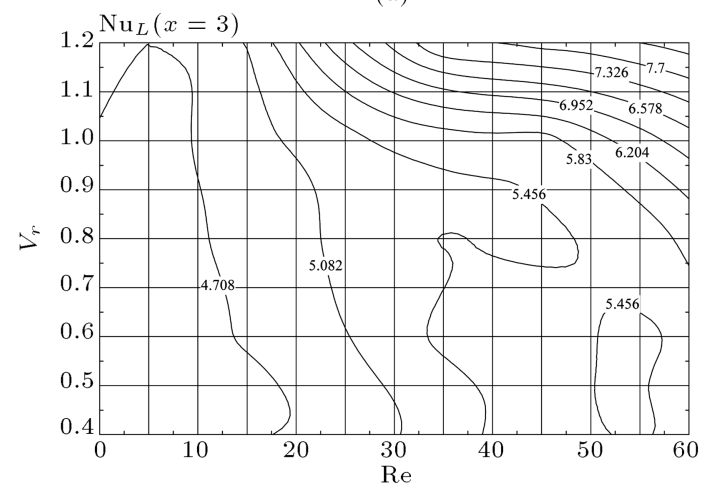

(c)

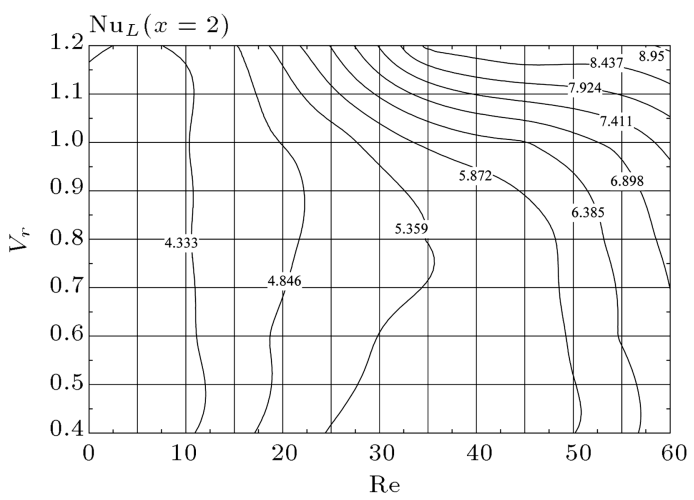

(b)

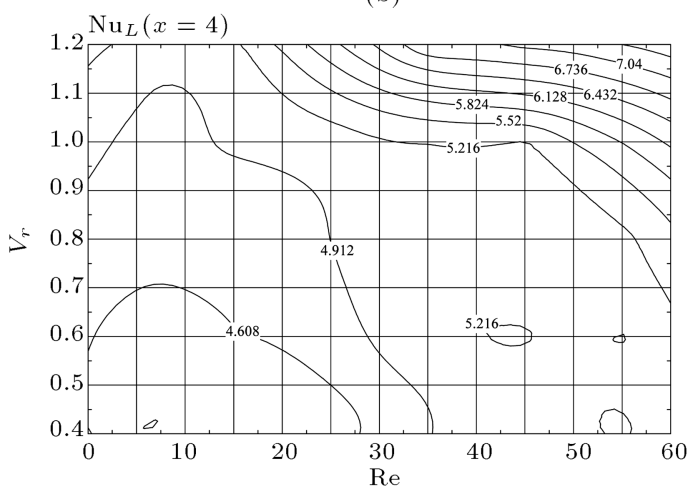

(d)

Figure 10. Generalized diagram of developing lower Nusselt number of stratified air-water flow as a function of the volumetric flow rate ratio and Reynolds number at $x=1,2,3$, and 4 from channel inlet. 


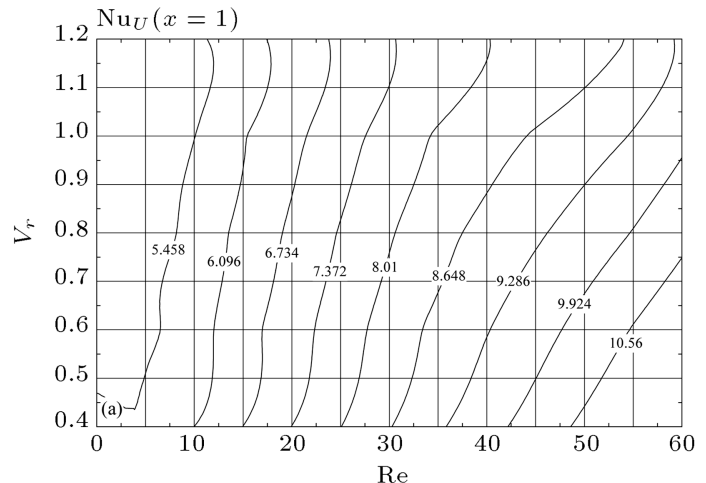

(a)

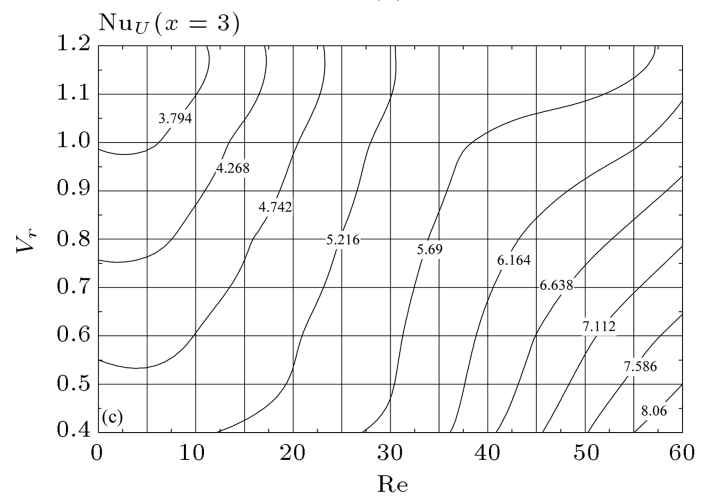

(c)

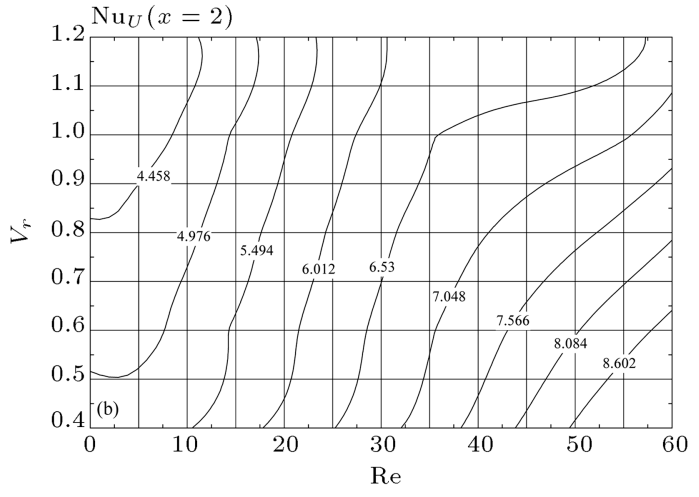

(b)

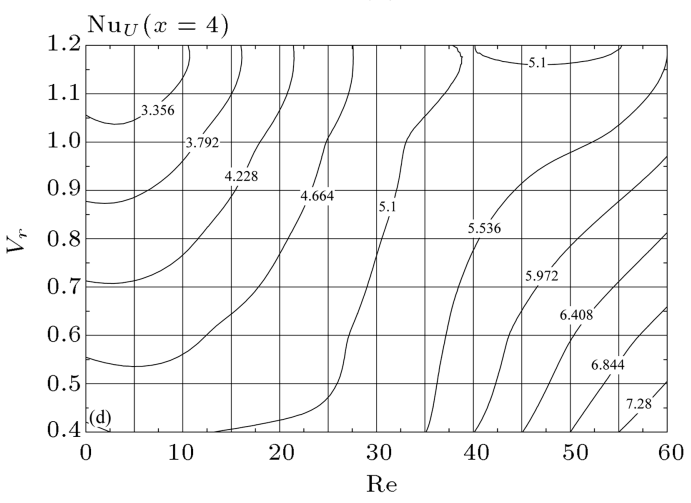

(d)

Figure 11. Generalized diagram of developing upper Nusselt number of stratified air-water flow as a function of the volumetric flow rate ratio and Reynolds number at $x=1,2,3$, and 4 from channel inlet.

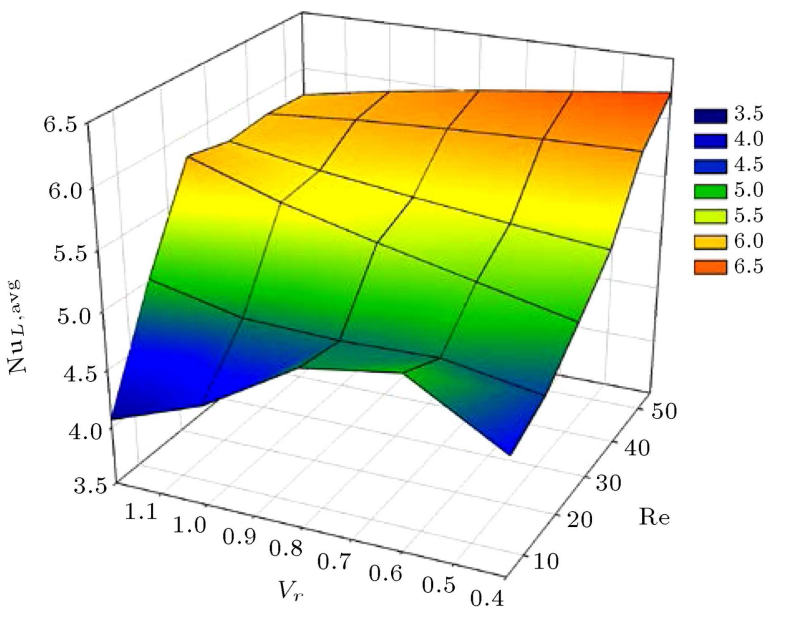

(a)

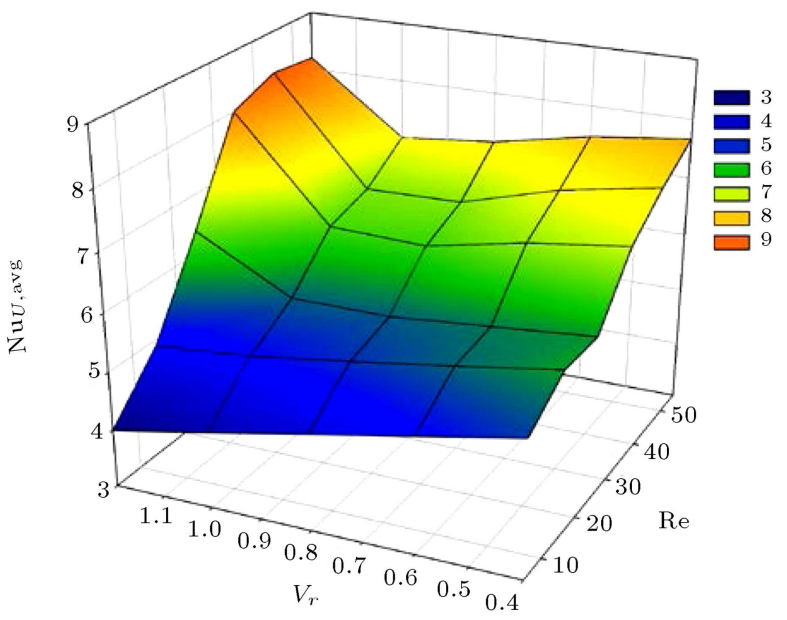

(b)

Figure 12. Generalized diagram of average Nusselt numbers of stratified air-water flow as a function of the volumetric flow rate ratio and Reynolds number: (a) Lower average Nusselt number, and (b) upper average Nusselt number.

occur far from the inlet; therefore, higher Nusselt numbers are observed by enhancing the Reynolds number. A decrease in the volumetric flow rate ratio increases the difference between the lower wall temperature and the bulk temperature in the lower fluid, which imposes a reduction in the lower Nusselt number.

In Figure 11, the generalized diagram of the upper Nusselt number is shown. By decreasing the volumetric flow rate ratio, enhancement of the upper Nusselt number is observed, which is because of reduction in the difference of the upper wall temperature and the total bulk temperature.

Average Nusselt number is one of the favorite output results of the studies dealing with heat transfer analysis. In Figure 12, the average upper and lower Nusselt numbers in the whole mini-channel are 
presented. As shown in Figure 12(a), in a Reynolds number equal to 5.0, an optimum value for the volumetric flow rate ratio is observed, which maximizes the average lower Nusselt number. The same phenomenon happens for a volumetric flow rate ratio of 1.2 in a specific Reynolds number. In Figure 12(b), the average upper Nusselt number is presented as a function of the Reynolds number as well as the volumetric flow rate ratio. If heat transfer enhancement is the objective of the design, in a Reynolds number of 50.0, the volumetric flow rate ratio of 1.0 should be avoided because it minimizes the average upper Nusselt number. Figure 12 reveals the importance of the parameter selection in the air-water stratified flow in a minichannel.

\subsection{Total entropy generation}

The total entropy generation is calculated by integration of the local entropy generation in channel domain as stated in Eq. (17) [9]:

$$
S_{\text {gen,total }}=\iint S_{\text {gen }}(x, y,) d x d y .
$$

Total entropy generation in the whole mini-channel is illustrated in Figure 13. It can be seen that in each volumetric flow rate ratio, a specific Reynolds number maximizes the total entropy generation. The reason of this phenomenon is extensively explained by Zehtabiyan-Rezaie et al. [9] .

Figures 12 and 13 should be utilized in parameter selection, simultaneously, since the heat transfer and total entropy generation are both very important in the mechanical systems.

\section{Conclusion}

This study presents generalized diagrams for some of the most important characteristics of the stratified

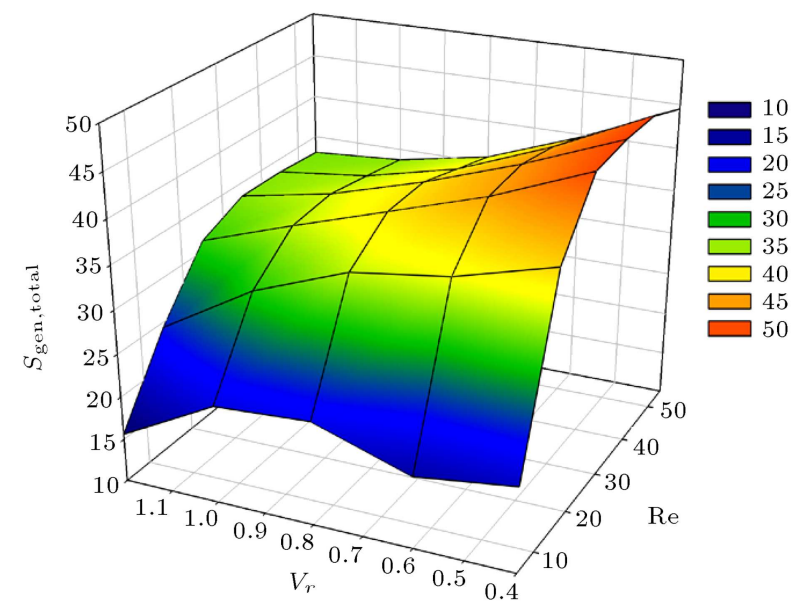

Figure 13. Generalized diagram of total entropy generation of stratified air-water flow as a function of the volumetric flow rate ratio and Reynolds number. air-water flow, heat transfer, and entropy generation in mini-channel by using a rule-based fuzzy inference system. Result analysis demonstrates that the system is an effective tool to avoid the considerable computational costs of the numerical codes. The main novelty of the study lies in the presentation of the generalized diagrams, which enhance the applicability of the results by other researchers. In a Reynolds number equal to 5.0, an optimum value for the volumetric flow rate ratio was observed, which maximized the average lower Nusselt number. The same phenomenon happened for a volumetric flow rate ratio of 1.2 in a specific Reynolds number. In addition, in a Reynolds number of 50.0, the volumetric flow rate ratio of 1.0 minimized the average upper Nusselt number. Another interesting result of the study is the generalized diagram of the total entropy generation, which reveals that a careful analysis should be applied in selection of the Reynolds number as well as the volumetric flow rate ratio.

\section{Nomenclature}

$c_{p}$

Number of clusters

Dimensionless specific heat at constant pressure

$D \quad$ Mini-channel height (m)

$D_{i k} \quad$ Mahalanobis distance

$f \quad$ Volume fraction

$F_{i} \quad$ Cluster covariance matrices

$h \quad$ Heat transfer coefficient $\left(\mathrm{W} / \mathrm{m}^{2} . \mathrm{K}\right)$

$J_{\mathrm{GK}} \quad$ The objective function of the GK algorithm

$k$

$L$

Dimensionless thermal conductivity

$L \quad$ Mini-channel length (m)

$m \quad$ Weighting exponent

$N \quad$ Number of data

$\mathrm{Nu} \quad$ Nusselt number

$P \quad$ Dimensionless pressure

Pr Prandtl number

$q^{\prime \prime} \quad$ Heat flux $\left(\mathrm{W} / \mathrm{m}^{2}\right)$

$q_{r}^{\prime \prime}=\frac{q_{U}^{\prime \prime}}{q_{L}^{\prime \prime}} \quad$ Heat flux ratio

Re Reynolds number

$S \quad$ Dimensionless entropy

$t \quad$ Dimensionless time

$T \quad$ Dimensionless temperature

$u \quad$ Dimensionless axial velocity

$u_{i k} \quad$ Cluster membership function

$v \quad$ Dimensionless normal velocity

$v_{i} \quad$ Cluster prototype 


$\begin{array}{ll}V_{r}=\frac{\dot{V}_{U}}{\dot{V}_{L}} & \text { Volumetric flow rate ratio } \\ \dot{V} & \text { Dimensionless volumetric flow rate } \\ \vec{V} & \text { Dimensionless velocity field } \\ x & \text { Dimensionless axial distance from inlet } \\ y & \text { Dimensionless normal distance from } \\ y^{*} & \text { lower wall } \\ & \text { Defuzzified output }\end{array}$

\section{Greek symbols}

$\alpha \quad$ Thermal diffusivity $\left(\mathrm{m}^{2} / \mathrm{s}\right)$

$\beta \quad$ Mamdani's Logical method combination parameter

$\delta \quad$ Dimensionless parameter in entropy generation equation

$\mu \quad$ Dimensionless viscosity

$\nu \quad$ Kinematic viscosity $\left(\mathrm{m}^{2} / \mathrm{s}\right)$

$\rho \quad$ Dimensionless density

$\omega \quad$ Yager operators parameter

$\xi \quad$ Dimensionless parameter in entropy generation equation

$\zeta \quad$ FATI-FITA combination parameter

\section{Subscripts}

$\begin{array}{ll}\text { avg } & \text { Average } \\ \text { bulk } & \text { Bulk temperature of fluid } \\ \text { F.D } & \text { Fully Developed } \\ \text { gen } & \text { Generation } \\ \text { in } & \text { Inlet } \\ i & \text { Interface } \\ L & \text { Lower fluid } \\ r & \text { Ratio of upper fluid properties to lower } \\ & \text { fluid properties } \\ U & \text { Upper fluid }\end{array}$

\section{Superscripts}

I

II!

Dimensional array variables

Per volume

Rate form

\section{References}

1. Collier, J.G. and Thome, J.R., Convective Boiling and Condensation, 3rd Edn., Clarendon Press, Oxford (1994).

2. Ishii, M., Thermo-Fluid Dynamic Theory of Two-phase Flow, Eyrolles, France (1975).

3. Ishii, M. and Hibiki, T., Thermo-Fluid Dynamics of Two-Phase Flow, Springer Science \& Business Media (2010).
4. Kolev, N.I., Multiphase Flow Dynamics 1: Fundamentals, Springer (2007).

5. Hansen, E.A. and Vested, H.J. "Liquid hold-up, pressure drop, and velocity profiles in steady uniform stratified flow", Journal of Energy Resources Technology, 113, pp. 87-93 (1991).

6. Yap, Y.F., Chai, J.C., Toh, K.C., Wong, T.N. and Lam, Y.C. "Numerical modeling of unidirectional stratified flow with and without phase change", International Journal of Heat and Mass Transfer, 48, pp. 477-486 (2005).

7. Datta, D., Gada, V.H. and Sharma, A. "Analytical and level-set method-based numerical study for twophase stratified flow in a plane channel and a square duct", Numerical Heat Transfer, Part A: Applications, 60, pp. 347-380 (2011).

8. Gada, V.H., Datta, D. and Sharma, A. "Analytical and numerical study for two-phase stratified-flow in a plane channel subjected to different thermal boundary conditions", International Journal of Thermal Sciences, 71, pp. 88-102 (2013).

9. Zehtabiyan-Rezaie, N., Saffar-Avval, M. and Mirzaei, M. "Analytical and numerical investigation of heat transfer and entropy generation of stratified two-phase flow in mini-channel", International Journal of Thermal Sciences, 90, pp. 24-37 (2015).

10. Hakeem, M., Kamil, M. and Arman, I. "Prediction of temperature profiles using artificial neural networks in a vertical thermosiphon reboiler", Applied Thermal Engineering, 28, pp. 1572-1579 (2008).

11. Kalogirou, S.A., Panteliou, S. and Dentsoras, A. "Artificial neural networks used for the performance prediction of a thermosiphon solar water heater", Renewable Energy, 18, pp. 87-99 (1999).

12. Karami, A., Yousefi, T., Ebrahimi, S., Rezaei, E. and Mahmoudinezhad, S. "Adaptive neuro-fuzzy inference system (ANFIS) to predict the forced convection heat transfer from a v-shaped plate", Heat and Mass Transfer, 49, pp. 789-798 (2013).

13. Mehrabi, M. and Pesteei, S.M. "Adaptive neuro-fuzzy modeling of convection heat transfer of turbulent supercritical carbon dioxide flow in a vertical circular tube", International Communications in Heat and Mass Transfer, 37, pp. 1546-1550 (2010).

14. Rosa, E.S., Salgado, R.M., Ohishi, T. and Mastelari, N. "Performance comparison of artificial neural networks and expert systems applied to flow pattern identification in vertical ascendant gas-liquid flows", International Journal of Multiphase Flow, 36, pp. 738754 (2010).

15. Abolbashari, M.H., Freidoonimehr, N., Nazari, F. and Rashidi, M.M. "Entropy analysis for an unsteady MHD flow past a stretching permeable surface in nanofluid", Powder Technology, 267, pp. 256-267 (2014).

16. Abolbashari, M.H., Freidoonimehr, N., Nazari, F. and Rashidi, M.M. "Analytical modeling of entropy generation for Casson nano-fluid flow induced by a 
stretching surface", Advanced Powder Technology, 26, pp. 542-552 (2015).

17. Gustafson, D.E. and Kessel, W.C., Fuzzy Clustering with a Fuzzy Covariance Matrix, Scientific Systems. Inc., Cambridge, MA (1978).

18. Sugeno, M. and Yasukawa, T. "A fuzzy-logic-based approach to qualitative modeling", IEEE Transactions on Fuzzy Systems, 1, pp. 7-31 (1993).

19. Bejan, A., Convection Heat Transfer, 3rd Edn., John Wiley and Sons Inc, Hoboken, New Jersey (2004).

20. Chorin, A.J. "Numerical solution of the Navier-Stokes equations", Mathematics of Computation, 22, pp. 745762 (1968).

21. Hirt, C.W. and Nichols, B.D. "Volume of fluid (VOF) method for the dynamics of free boundaries", Journal of Computational Physics, 39, pp. 201-225 (1981).

22. Ketabdari, M.J. and Saghi, H. "A Novel algorithm of advection procedure in volume of fluid method to model free surface flows", ISRN Applied Mathematics, 2012, p. 16 (2012).

23. Nobari, M.R.H., Ketabdari, M.J. and Moradi, M. "A modified volume of fluid advection method for uniform Cartesian grids", Applied Mathematical Modelling, 33, pp. 2298-2310 (2009).

24. Rider, W.J. and Kothe, D.B. "Reconstructing volume tracking", Journal of Computational Physics, 141, pp. 112-152 (1998).

25. Welch, S.W.J. and Wilson, J. "A volume of fluid based method for fluid flows with phase change", Journal of Computational Physics, 160, pp. 662-682 (2000).

26. Zadeh, L.A. "Fuzzy sets", J. Inf. Control, 8, pp. 338353 (1965).

27. Zarandi, M.H.F., Avazbeigi, M. and Türksen, I.B. "A new fuzzy noise-rejection data partitioning algorithm with revised mahalanobis distance", IFSA/EUSFLAT Conf., pp. 1520-1526 (2009).

28. Wu, K.L. and Yang, M.S. "A cluster validity index for fuzzy clustering", Pattern Recognition Letters, 26, pp. 1275-1291 (2005).

\section{Biographies}

Navid Zehtabiyan-Rezaie received the BSc and MSc degrees in Mechanical Engineering from Amirkabir University of Technology (AUT), Tehran, Iran, in 2011 and 2013, respectively. He is currently PhD candidate in Mechanical Engineering and Research Assistant at Amirkabir University of Technology. His research interests are heat transfer enhancement, entropy generation minimization, two-phase flow modeling, Computational Fluid Dynamics (CFD), and electro-hydrodynamics (EHD).

Saeede Rahimi Damirchi-Darasi received the BSc and MSc degrees in Industrial Engineering from Amirkabir University of Technology, Tehran, Iran, in 2012 and 2014, respectively. Her research interests are expert systems, artificial engineering, healthcare, and fuzzy logic systems.

Mohammad Hossein Fazel Zarandi is Professor in the Department of Industrial Engineering at Amirkabir University of Technology, Tehran, Iran, and a member of the Knowledge-Information Systems Laboratory of the University of Toronto, Canada. His main research interests focus on intelligent information systems, soft computing, computational intelligence, fuzzy sets and systems, multi-agent systems, networks, meta-heuristics, and optimization. Professor Fazel Zarandi has authored several books, scientific papers, and technical reports in the above areas, most of which are accessible on the web. He has also taught several courses in fuzzy systems engineering, decision support systems, management information systems, artificial intelligence and expert systems, systems analysis and design, scheduling, neural networks, simulations, and production planning and control at several universities in Iran and North America.

Majid Saffar-Avval is a Professor of Mechanical Engineering at Amirkabir University of Technology (AUT), Tehran, Iran. He received his BSc and MSc degrees from Sharif University of Technology and his PhD degree from the Ecole Nationale des Arts et Metiers (ENSAM), Paris, France, in 1985. He has been teaching at AUT since then. He was head of the Mechanical Engineering Department from June 2000 to June 2002 and head of the Energy and Control Center of Excellence from May 2007 to March 2012 at AUT. His research contributions are in the fields of two-phase heat transfer, advanced thermal systems, energy management, and bio-heat transfer. 